\title{
Neo-Timm staining in the thalamus of chronically epileptic rats
}

C. Hamani, I. de Paulo and L.E.A.M. Mello
Departamento de Fisiologia, Escola Paulista de Medicina, Universidade Federal de São Paulo, São Paulo, SP, Brasil

\author{
Correspondence \\ C. Hamani \\ Setor de Neurofisiologia \\ Departamento de Fisiologia \\ EPM, UNIFESP \\ R. Botucatu, 862 \\ 04023-062 São Paulo, SP \\ Brasil \\ Fax: +55-11-5579-2033 \\ E-mail: chamani@ig.com.br \\ Research supported by PRONEX, \\ Instituto do Milênio-CNPq, \\ FAPESP-CEPID and FAPESP.
}

Received October 26, 2004

Accepted June 10, 2005

$\ldots \ldots \ldots \ldots \ldots \ldots \ldots$

\begin{abstract}
The thalamus is an important modulator of seizures and is severely affected in cholinergic models of epilepsy. In the present study, chronically epileptic rats had their brains processed for neo-Timm and acetylcholinesterase two months after the induction of status epilepticus with pilocarpine. Both controls and pilocarpine-treated animals presented neo-Timm staining in the anterodorsal nucleus, laterodorsal nucleus, reticular nucleus, most intralaminar nuclei, nucleus reuniens, and rhomboid nucleus of the thalamus, as well as in the zona incerta. The intensity of neo-Timm staining was similar in control and pilocarpine-treated rats, except for the nucleus reuniens and the rhomboid nucleus, which had a lower intensity of staining in the epileptic group. In animal models of temporal lobe epilepsy, zinc seems to modulate glutamate release and to decrease seizure activity. In this context, a reduction of neo-Timm-stained terminals in the midline thalamus could ultimately result in an increased excitatory activity, not only within its related nuclei, but also in anatomical structures that receive their efferent connections. This might contribute to the pathological substrate observed in chronic pilocarpine-treated epileptic animals.
\end{abstract}

Key words

- Epilepsy

- Pilocarpine

- Zinc

- Sprouting

- Nucleus reuniens

- Midline thalamus

\section{Introduction}

Despite the role played by the thalamus in the circuitry of seizure activity, reorganization of epileptic pathways has mostly been studied in the hippocampus (1-3). After an epileptogenic insult, granule cell axons (mossy fibers) reorganize and establish an abnormal recurrent circuit that ultimately reinnervates the granule cell layer (1-3). Since mossy fibers are rich in zinc, the sprouting of this pathway has been recognized by the Timm technique (1-3). The Timm staining method and its variants detect mostly the chelatable pool of zinc that is concentrated in synaptic vesicles of terminal boutons (4-8). Even though hippocampal regions that receive mossy fiber terminals are the most robustly stained areas in the brain, Timmstained terminals have also been recognized in the amygdala, cortex, basal forebrain, and thalamus (7,9-11).

Anatomical and physiological studies suggest that several thalamic nuclei are involved in the mechanisms of epileptogenesis and seizure modulation in both animal models and human epilepsy (12-23). Yet, there is a lack of studies assessing circuitry reorgani- 
zation in the thalamus of epileptic animals. Since the pattern of neo-Timm staining has been recently detailed in the thalamus of normal rats (11), our objective here was to investigate whether thalamic zinc-rich terminals also present sprouting or reorganization in epileptic animals. Thus, we compared the pattern of neo-Timm staining in the thalamus of chronically epileptic rats treated with pilocarpine and controls.

\section{Material and Methods}

\section{Pilocarpine administration and status epilepticus}

All protocols were carried out in accordance with the Declaration of Helsinki and the Guide for the Care and Use of Laboratory Animals adopted and promulgated by the National Institutes of Health. The protocol used for induction of status epilepticus (SE) and spontaneous recurrent seizures after pilocarpine treatment has been previously described in detail (3). Briefly, adult male Wistar rats (150-250 g) were injected with methylscopolamine $(1 \mathrm{mg} / \mathrm{kg}$, ip $)$ followed $30 \mathrm{~min}$ later by pilocarpine $(320 \mathrm{mg} /$ $\mathrm{kg}$, ip). Approximately 20-30 min after pilocarpine administration the animals developed SE, characterized by the occurrence of continuous behavioral seizures. Ninety minutes after SE onset, the animals were injected with thionembutal $(25 \mathrm{mg} / \mathrm{kg}$, ip $)$ to reduce the otherwise high mortality rate observed during this period. For the 2-3 subsequent days, the animals received oral saline and sucrose as well as subcutaneous $10 \%$ glucose in $0.9 \%$ saline supplements. Three to four weeks after SE, they developed spontaneous recurrent seizures and were characterized as chronically epileptic animals.

\section{Histologic procedures}

One hundred and twenty days after SE, epileptic animals $(\mathrm{N}=5)$ were injected with sodium selenite $(15 \mathrm{mg} / \mathrm{kg}$, ip $)$ in order to enhance neo-Timm staining (7). Two hours later, they were deeply anesthetized with thionembutal $(50 \mathrm{mg} / \mathrm{kg}$, ip $)$ and transcardially perfused with i) $25 \mathrm{ml}$ Millonig's buffer, ii) $50 \mathrm{ml} 0.1 \% \mathrm{Na}_{2} \mathrm{~S}$ in Millonig' s buffer, iii) $100 \mathrm{ml} \mathrm{3 \%}$ glutaraldehyde, and iv) $200 \mathrm{ml}$ $0.1 \% \mathrm{Na}_{2} \mathrm{~S}$ in Millonig's buffer. Next, 40$\mu \mathrm{m}$ thick coronal sections were cut with a cryostat and developed according to standard neo-Timm protocols in a solution containing arabic gum, citrate buffer, hydroquinone, and silver nitrate, for 60-70 $\mathrm{min}$ in the dark at $26^{\circ} \mathrm{C}$ (3). Adjacent sections were processed for acetylcholinesterase (AChE) activity. Age-matched naive male Wistar rats $(\mathrm{N}=5)$ had their brains processed according to the same protocols and were used as the control group.

\section{Intensity of neo-Timm staining}

All neo-Timm-stained thalamic nuclei were evaluated. The epithalamus was not assessed. neo-Timm staining was scored as follows: 0 , no staining; + , very lightly stained; ++ lightly stained; +++, moderately stained; ++++, densely stained. Adjacent AchEstained sections were used to visualize the internuclear thalamic boundaries in epileptic and control animals (11). Cresyl violet was not used for this purpose due to its limited precision in delineating the borders of thalamic nuclei in gliotic tissue.

\section{Results}

\section{Neo-Timm-stained thalamic nuclei and zona} incerta

The following nuclei showed neo-Timm staining in both control and epileptic animals: anterodorsal (AD), laterodorsal (LD), reticular (Rt), central medial $(\mathrm{CM})$, paracentral (PC), central lateral (CL), reuniens ( $\mathrm{Re})$, and rhomboid (Rh). In addition, we also observed neo-Timm staining in the zona 
incerta. Even though this structure comprises part of the subthalamic region and is not a formal thalamic nucleus, we decided to include it in our analysis.

\section{Intensity and pattern of neo-Timm staining}

The intensity of neo-Timm staining in almost all the thalamic nuclei and in the zona incerta was similar in pilocarpine-treated and control animals (Table 1).

The AD nucleus was densely stained, particularly in its dorsolateral region. The LD nucleus presented a variable degree of injury in different epileptic animals. In general, the portions of the LD that were not gliotic showed dense neo-Timm staining (Figure 1). In epileptic animals with intense tissue damage, the internuclear boundary between $\mathrm{LD}$ and the latero-posterior nucleus presented pathologic abnormalities similar to the calcifications previously reported for chronically epileptic rats treated with pilocarpine and picrotoxin (Figure 1E,F) (14). The Rt nucleus was densely stained in its most rostral and dorsal portions. Caudal and ventral regions of the Rt were either very lightly stained or not stained at all. Intralaminar nuclei, such as the CM, PC and CL nuclei, showed moderate neo-Timm staining. Neo-Timm staining was more prominent in the rostral levels of CM and PC and in the caudal levels of CL nuclei. The zona incerta presented a moderate staining pattern, most prominent in the rostral levels of its ventral region.

Contrasting with the previously described nuclei, the $\mathrm{Re}$ and $\mathrm{Rh}$ nuclei showed a lower intensity of neo-Timm staining in the epileptic group compared to control. While the Re was lightly to moderately stained and the $\mathrm{Rh}$ was lightly stained in control animals (Figure $2 \mathrm{~B}$ ), both nuclei were only very lightly stained in pilocarpine-treated rats (Figure 2D). Even though the cytoarchitecture of these structures was compromised in the epileptic animals, both Re and Rh could still
Table 1. Intensity of neo-Timm staining in the zona incerta and thalamus of controls and chronically epileptic animals treated with pilocarpine.

\begin{tabular}{lcc}
\hline & Control & Pilocarpine \\
\hline Anterodorsal nucleus & ++++ & ++++ \\
Laterodorsal nucleus & +++++++ & $+++/++++$ \\
Reticular nucleus (rostrodorsal region) & ++++ & ++++ \\
Reticular nucleus (caudal-ventral region) & $0 /+$ & $0 /+$ \\
Central medial nucleus & +++ & +++ \\
Paracentral nucleus & +++ & +++ \\
Central lateral nucleus & +++ & +++ \\
Zona incerta & +++ & +++ \\
Nucleus reuniens & ++++++ & + \\
Rhomboid nucleus & ++ & +
\end{tabular}

$\mathrm{N}=$ five animals per group. 0 , no staining; +, very lightly stained; ++, lightly stained; +++ , moderately stained; ++++, densely stained. See Material and Methods for treatment with pilocarpine.
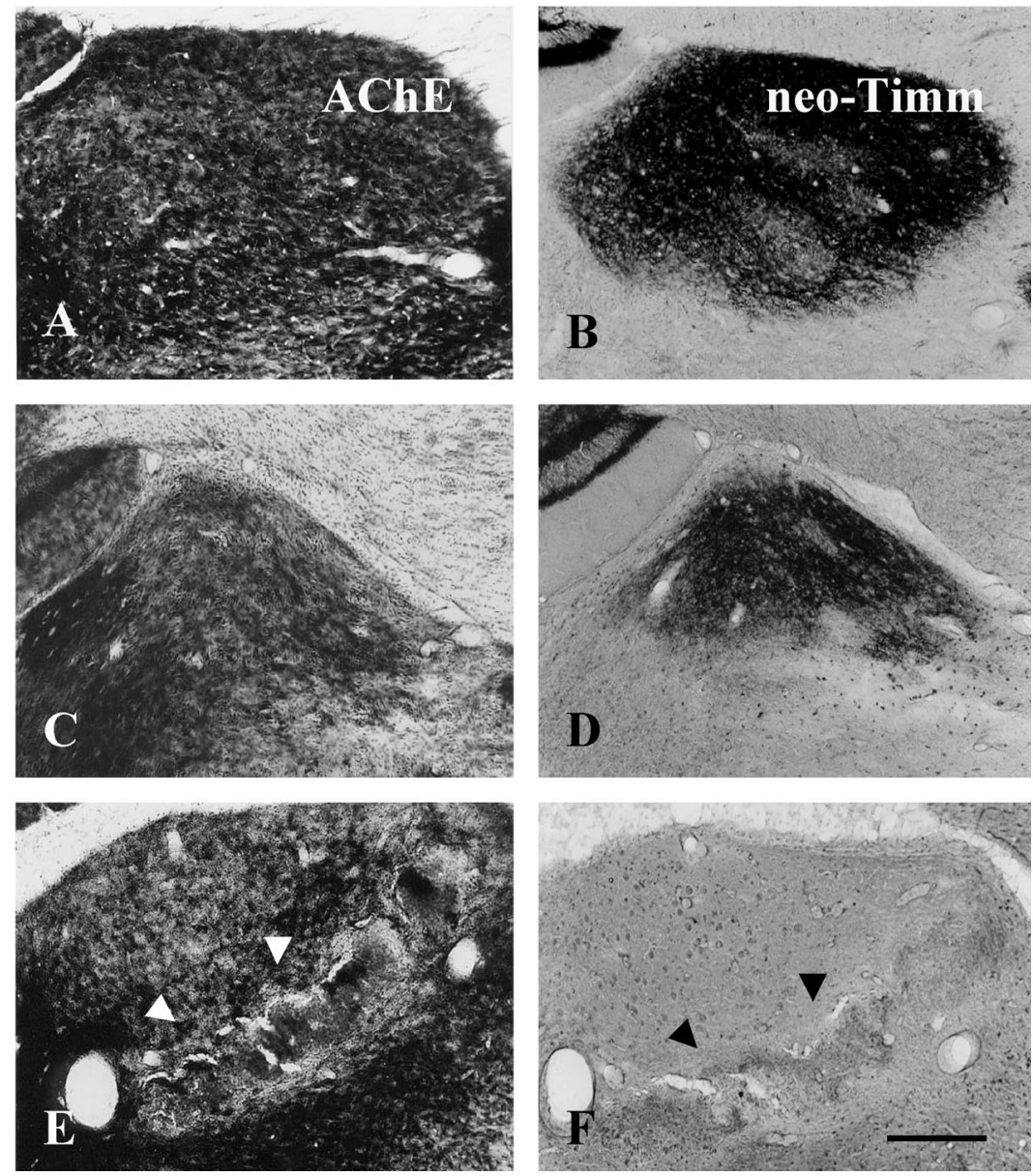

Figure 1. Acetylcholinesterase (AChE) and neo-Timm staining of the laterodorsal nucleus of the thalamus in a control $(A, B)$ and two chronically epileptic rats treated with pilocarpine as described in Material and Methods (C,D,E,F). Pathology was not homogeneous within this nucleus. While some of the animals had a relatively preserved cytoarchitecture $(C, D)$, others had more severe alterations, including pathologic calcifications in the boundary region between the laterodorsal nucleus and the lateroposterior nucleus (arrowheads; E,F). Scale bar $=500 \mu \mathrm{m}$. 
be delineated in AChE sections (Figure $2 \mathrm{~A}, \mathrm{C})$. This indicates that the decrease in neo-Timm staining seen in these nuclei was probably related to specific pathologic events and not merely a consequence of the volumetric changes seen in the epileptic group.

We did not find evidence of sprouting in any of the thalamic nuclei assessed.

\section{Discussion}

We have shown here that 1) several thalamic nuclei and the zona incerta stained for zinc in both control and pilocarpine-treated rats, and 2) the intensity of neo-Timm staining was decreased in the Re and Rh nuclei in chronically epileptic animals.

Almost all thalamic nuclei that stained for neo-Timm in our study, as well as the zona incerta, have been previously implied in mechanisms of epileptogenesis or seizure modulation. Lesions, high frequency stimulation, or the pharmacological blockage of the anterior nucleus reduce the propensity for seizures in animal models and in patients with epilepsy $(15-17,20)$. In contrast, phar-
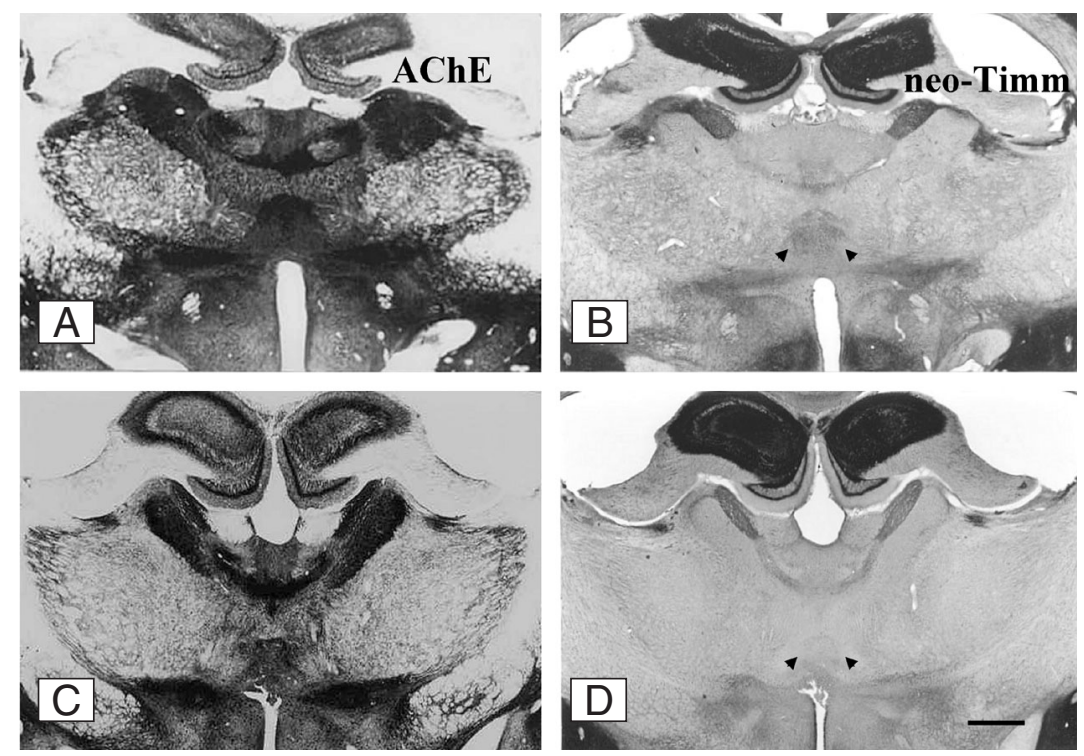

Figure 2. Acetylcholinesterase (AChE) and neo-Timm staining of the thalamus in a control $(A, B)$ and a chronically epileptic rat treated with pilocarpine as described in Material and Methods (C,D). Note the decreased intensity of neo-Timm staining in the region of the rhomboid nucleus and nucleus reuniens in the pilocarpine-treated animal (arrowheads). Scale bar $=2 \mathrm{~mm}$. macological studies have suggested that the zona incerta might be involved in inhibitory circuits of epileptogenesis in the pilocarpine model of epilepsy (24). The reticular nucleus of the thalamus and the rostral intralaminar group have been involved in the mechanisms of thalamo-cortical recruiting rhythms, spike and wave discharge, and the pathophysiology of absence seizures $(21,23,25)$. The topical administration of GABAergic and cholinergic agents to the region of the $\mathrm{CM}$ nucleus influenced seizure activity in the pentylenetetrazole model of epilepsy $(18,19)$.

The $\mathrm{Re}$ and $\mathrm{Rh}$ nuclei comprise part of the so-called midline thalamus and have been involved in mechanisms of seizure activity in animal models of limbic epilepsy (12). Midline thalamic activity was noticed at early stages of seizure evolution during hippocampal kindling and in chronically epileptic rats (12). Moreover, chronically epileptic animals develop changes in electrophysiological properties, such as synaptically mediated and voltage-gated responses (12), as well as neuronal loss and gliosis in both $\mathrm{Re}$ and $\mathrm{Rh}(12,14)$. These pathological substrates and the important anatomical connections between the Re and the hippocampus may be responsible in part for the enhanced excitability of thalamo-limbic pathways in epileptic circuits $(12,26,27)$. Reuniens axons form asymmetrical synapses on spines and dendrites of pyramidal cells and interneurons and Re stimulation depolarizes pyramidal cells and evokes spiking activity in the oriens-alveus and radiatum strata (12,26-30). Excitatory amino acids seem to be the putative neurotransmitters of Re-hippocampal pathways (31).

The role of zinc as a neuromodulator in epilepsy is controversial. Depending on the animal model, the receptor type and subunit configuration, zinc can act as a pro- or anticonvulsant agent (32-35). In animal models of temporal lobe epilepsy, however, zinc seems to decrease seizure activity (34-36). 
Mice lacking vesicular zinc and dietary zincdeficient rats are not only more susceptible to kainic acid-induced seizures, but also have a higher degree of neuronal injury in the hippocampus $(34,35)$. Whether the neuroprotective effects of zinc derive from a reduced release of glutamate or an increase in the concentration of GABA remains elusive, since both mechanisms have been demon- strated in microdialysis studies (37-39). Yet, regardless of the mechanism involved, the reduction of Timm-stained terminals observed in the midline thalamus in our study could ultimately result in an increased excitatory activity within the Re and Rh, damaging not only their own cytoarchitecture but also that of anatomical structures that receive their efferent connections (40).

\section{References}

1. Sutula T, Cascino G, Cavazos J et al. (1989). Mossy fiber synaptic reorganization in the epileptic human temporal lobe. Annals of Neurology, 26: 321-330.

2. Tauck DL \& Nadler JV (1985). Evidence of functional mossy fiber sprouting in hippocampal formation of kainic acid-treated rats. Journal of Neuroscience, 5: 1016-1022.

3. Mello LE, Cavalheiro EA, Tan AM et al. (1993). Circuit mechanisms of seizures in the pilocarpine model of chronic epilepsy: cell loss and mossy fiber sprouting. Epilepsia, 34: 985-995.

4. Danscher G (1982). Exogenous selenium in the brain. A histochemical technique for light and electron microscopical localization of catalytic selenium bonds. Histochemistry, 76: 281-293.

5. Danscher G (1981). Histochemical demonstration of heavy metals. A revised version of the sulphide silver method suitable for both light and electronmicroscopy. Histochemistry, 71: 1-16.

6. Danscher G, Howell G, Perez-Clausell J et al. (1985). The dithizone, Timm's sulphide silver and the selenium methods demonstrate a chelatable pool of zinc in CNS. A proton activation (PIXE) analysis of carbon tetrachloride extracts from rat brains and spinal cords intravitally treated with dithizone. Histochemistry, 83: 419-422.

7. Slomianka L, Danscher G \& Frederickson CJ (1990). Labeling of the neurons of origin of zinc-containing pathways by intraperitoneal injections of sodium selenite. Neuroscience, 38: 843-854.

8. Perez-Clausell J \& Danscher G (1985). Intravesicular localization of zinc in rat telencephalic boutons. A histochemical study. Brain Research, 337: 91-98.

9. Christensen MK \& Geneser FA (1995). Distribution of neurons of origin of zinc-containing projections in the amygdala of the rat. Anatomical Embryology, 191: 227-237.

10. Frederickson CJ, Rampy BA, Reamy-Rampy $S$ et al. (1992). Distribution of histochemically reactive zinc in the forebrain of the rat. Journal of Chemical Neuroanatomy, 5: 521-530.

11. Mengual E, Casanovas-Aguilar C, Perez-Clausell $\mathrm{J}$ et al. (2001). Thalamic distribution of zinc-rich terminal fields and neurons of origin in the rat. Neuroscience, 102: 863-884.

12. Bertram EH, Mangan PS, Zhang D et al. (2001). The midline thalamus: alterations and a potential role in limbic epilepsy. Epilepsia, 42: 967-978.

13. Hamani C \& Mello LE (1997). Status epilepticus induced by pilocarpine and picrotoxin. Epilepsy Research, 28: 73-82.

14. Hamani C \& Mello LE (2002). Thalamic neuropathology in the chronic pilocarpine and picrotoxin model of epilepsy. Thalamus and Related Systems, 2: 49-53.

15. Hamani C, Ewerton FI, Bonilha SM et al. (2004). Bilateral anterior thalamic nucleus lesions and high-frequency stimulation are protective against pilocarpine-induced seizures and status epilepticus. Neurosurgery, 54: 191-195; discussion 195-197.

16. Hodaie M, Wennberg RA, Dostrovsky JO et al. (2002). Chronic anterior thalamus stimulation for intractable epilepsy. Epilepsia, 43: 603-608.

17. Kerrigan JF, Litt B, Fisher RS et al. (2004). Electrical stimulation of the anterior nucleus of the thalamus for the treatment of intractable epilepsy. Epilepsia, 45: 346-354.

18. Miller JW \& Ferrendelli JA (1990). Characterization of GABAergic seizure regulation in the midline thalamus. Neuropharmacology, 29: 649-655.

19. Miller JW, Gray BC \& Bardgett ME (1992). Characterization of cholinergic regulation of seizures by the midline thalamus. Neuropharmacology, 31: 349-356.

20. Mirski MA, Rossell LA, Terry JB et al. (1997). Anticonvulsant effect of anterior thalamic high frequency electrical stimulation in the rat. Epilepsy Research, 28: 89-100.

21. Snead 3rd OC (1995). Basic mechanisms of generalized absence seizures. Annals of Neurology, 37: 146-157.

22. Scorza FA, Sanabria ER, Calderazzo L et al. (1998). Glucose utilization during interictal intervals in an epilepsy model induced by pilocarpine: a qualitative study. Epilepsia, 39: 1041-1045.

23. Slaght SJ, Leresche N, Deniau JM et al. (2002). Activity of thalamic reticular neurons during spontaneous genetically determined spike and wave discharges. Journal of Neuroscience, 22: 2323-2334.

24. Hamani C, Sakabe S, Bortolotto ZA et al. (2002). Inhibitory role of the zona incerta in the pilocarpine model of epilepsy. Epilepsy Research, 49: 73-80.

25. Seidenbecher T \& Pape HC (2001). Contribution of intralaminar thalamic nuclei to spike-and-wave-discharges during spontaneous seizures in a genetic rat model of absence epilepsy. European Journal of Neuroscience, 13: 1537-1546.

26. Dolleman-Van der Weel MJ \& Witter MP (2000). Nucleus reuniens thalami innervates gamma aminobutyric acid positive cells in hippocampal field CA1 of the rat. Neuroscience Letters, 278: 145-148.

27. Dolleman-Van Der Weel MJ \& Witter MP (1996). Projections from the nucleus reuniens thalami to the entorhinal cortex, hippocampal field CA1, and the subiculum in the rat arise from different populations of neurons. Journal of Comparative Neurology, 364: 637-650.

28. Dolleman-Van der Weel MJ, Lopes da Silva FH \& Witter MP (1997). Nucleus reuniens thalami modulates activity in hippocampal field CA1 through excitatory and inhibitory mechanisms. Journal of Neuroscience, 17: 5640-5650. 
29. Wouterlood FG, Saldana E \& Witter MP (1990). Projection from the nucleus reuniens thalami to the hippocampal region: light and electron microscopic tracing study in the rat with the anterograde tracer Phaseolus vulgaris-leucoagglutinin. Journal of Comparative Neurology, 296: 179-203.

30. Bertram EH \& Zhang DX (1999). Thalamic excitation of hippocampal CA1 neurons: a comparison with the effects of CA3 stimulation. Neuroscience, 92: 15-26.

31. Bokor H, Csaki A, Kocsis K et al. (2002). Cellular architecture of the nucleus reuniens thalami and its putative aspartatergic/glutamatergic projection to the hippocampus and medial septum in the rat. European Journal of Neuroscience, 16: 1227-1239.

32. Mody I (1999). Synaptic plasticity in kindling. Advances in Neurology, 79: 631-643.

33. Williamson A \& Spencer D (1995). Zinc reduces dentate granule cell hyperexcitability in epileptic humans. NeuroReport, 6: 1562-1564.

34. Cole TB, Robbins CA, Wenzel HJ et al. (2000). Seizures and neuronal damage in mice lacking vesicular zinc. Epilepsy Research, 39: 153-169.
35. Takeda A, Hirate M, Tamano H et al. (2003). Susceptibility to kainateinduced seizures under dietary zinc deficiency. Journal of Neurochemistry, 85: 1575-1580.

36. Takeda A, Hirate M, Tamano $\mathrm{H}$ et al. (2003). Zinc movement in the brain under kainate-induced seizures. Epilepsy Research, 54: 123129

37. Westbrook GL \& Mayer ML (1987). Micromolar concentrations of $\mathrm{Zn}^{2+}$ antagonize NMDA and GABA responses of hippocampal neurons. Nature, 328: 640-643.

38. Takeda A, Minami A, Seki Y et al. (2003). Inhibitory function of zinc against excitation of hippocampal glutamatergic neurons. Epilepsy Research, 57: 169-174.

39. Takeda A, Minami A, Seki $Y$ et al. (2004). Differential effects of zinc on glutamatergic and GABAergic neurotransmitter systems in the hippocampus. Journal of Neuroscience Research, 75: 225-229.

40. Olney JW, Collins RC \& Sloviter RS (1986). Excitotoxic mechanisms of epileptic brain damage. Advances in Neurology, 44: 857877. 